Correspondence

Lígia M. Saraiva

Ist@itqb.unl.pt
Received 3 September 2008

Revised 2 December 2008

Accepted 8 December 2008

\section{Exploring the antimicrobial action of a carbon monoxide-releasing compound through whole-genome transcription profiling of Escherichia coli}

\author{
Lígia S. Nobre, ${ }^{1}$ Fátima Al-Shahrour, ${ }^{2}$ Joaquin Dopazo ${ }^{2,3,4}$ \\ and Lígia M. Saraiva ${ }^{1}$
}
${ }^{1}$ Instituto de Tecnologia Química e Biológica, Universidade Nova de Lisboa, Avenida da República (EAN), 2780-157 Oeiras, Portugal
${ }^{2}$ Department of Bioinformatics, Centro de Investigación Príncipe Felipe (CIPF), Valencia, E-46013, Spain
${ }^{3}$ Center for Biomedical Research on Rare Diseases (CIBERER), Centro de Investigación Príncipe Felipe (CIPF), Valencia, E-46013, Spain
${ }^{4}$ Functional Genomics Node (National Institute for Bioinformatics, INB), Centro de Investigación Príncipe Felipe (CIPF), Valencia, E-46013, Spain

\begin{abstract}
We recently reported that carbon monoxide (CO) has bactericidal activity. To understand its mode of action we analysed the gene expression changes occurring when Escherichia coli, grown aerobically and anaerobically, is treated with the CO-releasing molecule CORM-2 (tricarbonyldichlororuthenium(II) dimer). Microarray analysis shows that the E. coli CORM-2 response is multifaceted, with a high number of differentially regulated genes spread through several functional categories, namely genes involved in inorganic ion transport and metabolism, regulators, and genes implicated in post-translational modification, such as chaperones. CORM-2 has a higher impact in E. coli cells grown anaerobically, as judged by the repression of genes belonging to eight functional classes which are not seen in the response of aerobically CORM-2treated cells. The biological relevance of the variations caused by CORM- 2 was substantiated by studying the CORM-2 sensitivity of selected E. coli mutants. The results show that the deletion of redox-sensing regulators SoxS and OxyR increased the sensitivity to CORM-2 and suggest that while SoxS plays an important role in protection against CORM-2 under both growth conditions, OxyR seems to participate only in the aerobic CORM-2 response. Under anaerobic conditions, we found that the heat-shock proteins IbpA and IbpB contribute to CORM-2 defence since the deletion of these genes increases the sensitivity of the strain. The induction of several met genes and the hypersensitivity to CORM-2 of the $\Delta$ metR, $\Delta$ metl and $\Delta$ met $N$ mutant strains suggest that $\mathrm{CO}$ has effects on the methionine metabolism of $E$. coli. CORM-2 also affects the transcription of several $E$. coli biofilm-related genes and increases biofilm formation in $E$. coli. In particular, the absence of $\operatorname{tqs} A$ or bhs $A$ increases the resistance of $E$. coli to CORM-2, and deletion of tsqA leads to a strain that has lost its capacity to form biofilm upon treatment with CORM-2. In spite of the relatively stable nature of the $\mathrm{CO}$ molecule, our results show that $\mathrm{CO}$ is able to trigger a significant alteration in the transcriptome of $E$. coli which necessarily has effects in several key metabolic pathways.
\end{abstract}

\title{
INTRODUCTION
}

Abbreviations: CORM, CO-releasing molecule; CORM-2, tricarbonyldichlororuthenium(II) dimer; FDR, false discovery rate.

The GEO accession number for the microarray data associated with this paper is GSE13982.

Six supplementary tables are available with the online version of this paper.
Carbon monoxide $(\mathrm{CO})$ formed in nature results from the incomplete oxidation of organic matter and as the byproduct of the activity of the haem oxygenase enzyme, which oxidizes haem to biliverdin (Li et al., 2007). In the latter process, which can occur in bacteria, plants and animals, the amount of CO endogenously produced by the 
various cell types is low. In humans, the CO formed has several physiological functions in the neuronal and cardiovascular systems (Wu \& Wang, 2005). However, high concentrations of $\mathrm{CO}$ have a toxic effect in mammals due to the binding of $\mathrm{CO}$ to haemoglobin; the consequent impairment of oxygen storage and transport by haemoglobin causes hypoxia (Wu \& Wang, 2005). CO binds preferentially to transition-metal-containing proteins, including haem-based proteins/sensors, giving rise to structural modifications and alteration of their biological function which may also account for the toxic effects of CO. However, it has been shown that living cells can tolerate $\mathrm{CO}$ at the concentration of $0.01 \%$ (100 p.p.m.) for several hours (Otterbein \& Choi, 2000), and rodents could be exposed continuously for up to 2 years to 500 p.p.m. CO without deleterious effects (Otterbein \& Choi, 2000; Stupfel \& Bouley, 1970; Verma et al., 1993).

Following the discovery that carbon monoxide is physiologically important, molecules acting as CO-carriers, namely 'CO-releasing molecules' (CORMs), were developed. In order to mimic the role of $\mathrm{CO}$ derived from haem oxygenase (Ryter et al., 2006), these molecules have to be able to liberate $\mathrm{CO}$ in a biological environment. This liberation is triggered by some physical, chemical or enzymic stimulus. For many CORMs that carry molecular $\mathrm{CO}$ as a ligand to a central transition metal, the lack of a pharmacological effect of a similar compound devoid or depleted of $\mathrm{CO}$ has been taken as a positive proof for the biological action of $\mathrm{CO}$ (Dulak et al., 2002). Following this principle, the beneficial therapeutic action of several CORMs in a wide range of vasoactive and anti-inflammatory situations and models has been attributed to CO (Motterlini et al., 2005).

Recently, we reported that four transition-metal-based CORMs at micromolar concentrations have antibiotic activity, reducing the viability of bacteria such as Escherichia coli and Staphylococcus aureus, under both aerobic and anoxic conditions (Nobre et al., 2007). In that work we also showed that the bactericidal action of those CORMs was only effective when $\mathrm{CO}$ was taken up by the cells. In fact, we demonstrated that one of the CORMs led to extensive accumulation of the transition metal molybdenum inside bacterial cells. In all cases, the addition of haemoglobin to the medium, before addition of the CORM, prevented any bactericidal action. Separate experiments (Motterlini et al., 2002; J. D. Seixas, personal communication) showed that these CORMs transfer $\mathrm{CO}$ to haemoglobin at a very fast rate. Taken together these results strongly suggest that the bactericidal action of a given CORM depends on its ability to enter bacterial cells and enable $\mathrm{CO}$ to reach specific targets.

We have now extended these studies to the analysis of the global transcriptome of E. coli treated with CORM-2, which reflects the organism's immediate response to the environment. Additionally, we have examined the phenotype of 12 mutant strains to test the role of each deleted gene in the E. coli CORM-2 response. Regulatory genes and genes involved in methionine metabolism and biofilm formation were found to play an important role when the micro-organism is exposed to the bactericidal action of CORM-2.

\section{METHODS}

Bacterial strains and growth conditions. Escherichia coli MG1655 was grown, at $37{ }^{\circ} \mathrm{C}$ and 150 r.p.m., in minimal medium salts (MS) (Nobre et al., 2007) in the presence or absence of oxygen. Aerobic experiments were performed in flasks filled with $1 / 5$ of their volume and anaerobic experiments in rubber-sealed flasks that, once filled with medium and closed, were extensively flushed with nitrogen gas. Cultures were grown to an $\mathrm{OD}_{600}$ of 0.3 , and at this point cells were left untreated or treated with CORM-2 (Sigma).

RNA isolation. The hot-phenol method was used to extract total RNA from two independent cultures of cells grown either aerobically or anaerobically and treated with $250 \mu \mathrm{M}$ CORM-2 for $15 \mathrm{~min}$. The concentration of the drug added to the cultures is equivalent to 0.5 times the minimal inhibitory concentration of $500 \mu \mathrm{M}$ which was previously determined (Nobre et al., 2007). After the isolation procedure, RNA was incubated with DNase (Ambion), and once the absence of any residual DNA was confirmed, concentration and purity were determined in a Nanodrop ND-1000 UV-visible spectrophotometer. RNA integrity was checked in an Agilent 2100 Bioanalyser coupled to an RNA Nano-Assay (Agilent Technologies).

Microarray analysis. Total RNA $(10 \mu \mathrm{g})$ was processed for use in the Affymetrix GeneChip E. coli Genome 2.0 Arrays, according to the manufacturer's instructions. Arrays were scanned in an Affymetrix GeneChip scanner 2500 and analysed first with Affymetrix MAS 5.0 software to obtain Absent/Present calls and to ensure that all quality parameters were within the recommended range. Hybridization, scanning and detection procedures were done at the Genomics Unit of the Instituto Gulbenkian de Ciência (Portugal). The advantage of using the Affymetrix GeneChip is that it contains 20 probe pairs to detect each ORF, providing multiple independent measurements for each transcript. The subsequent analysis was performed with the dChip software program (Li \& Wong, 2001). The invariant set method (Li \& Hung Wong, 2001) was used to normalize arrays at the probe cell level for comparison purposes, and the model-base (Perfect Match-only model) was used to probe selection and computing expression values. The expression profiles of 4306 genes encoding proteins of E. coli K-12 MG155 were analysed (intergenic regions were not included) and genes were considered to have significant differential expression when they showed a transcriptional fold change $>2$ (with a $90 \%$ confidence interval) and a $P$-value $<0.05$ for a paired Student's $t$-test. To minimize occurrence of false positives the comparison criterion was carefully chosen to ensure that the empirical false discovery rate (FDR) was low (random permuting of the samples 200 times led to a median of FDR of $0 \%$ and $1.1 \%$ for the arrays acquired for cells grown anaerobically and aerobically, respectively).

Gene ontology analysis. To investigate the biological relevance of the differential gene expression profile, the microarray data were analysed with the FatiGO algorithm (Al-Shahrour et al., 2004, 2006). Briefly, FatiGO considers two lists of genes (in this case the genes upor downregulated by CORM-2 anaerobically or aerobically were compared with the unchanged genes) and converts them into two lists of gene ontology (GO) terms. Subsequently, FatiGO determines separately the percentage of the relative frequency of genes in each group that is annotated with a particular GO term (biological process or molecular function), and finally, a Fisher's exact test is used to 
determine if the GO term is significantly over- or under-represented in one of the groups with respect to the other one. Hence, it retrieves information about the predominant biological process or molecular function for the genes that are differently expressed in a certain experimental condition. In this study, the GO terms were considered differently represented when they exhibited a FDR-adjusted $P$-value $<0.2$. FatiGO is implemented in a web tool at: http://www.fatigo.org and within the Babelomics environment (http://www.babelomics.org).

Quantitative real-time RT-PCR. DNA microarray data were validated by quantitative real-time RT-PCR. To this end, $2.5 \mu \mathrm{g} \mathrm{E}$. coli total RNA, derived from two independent samples grown aerobically or anaerobically and treated with $250 \mu \mathrm{M}$ CORM-2 or with $500 \mu \mathrm{M} \mathrm{Ru}(\mathrm{II}) \mathrm{Cl}_{2}$ (DMSO) ${ }_{4}$, was used to synthesize cDNA, using the first-strand synthesis protocol of the Universal RiboClone cDNA Synthesis System (Promega). Each cDNA synthesis reaction was performed using $40 \mu \mathrm{g} \mathrm{ml}^{-1}$ of random primers, $40 \mathrm{U}$ RNasin RNase inhibitor, $4 \mathrm{mM}$ sodium pyrophosphate and $30 \mathrm{U}$ AMV reverse transcriptase. After amplification, cDNA was purified by phenol extraction and ethanol precipitation. Real-time PCRs were performed in a LightCycler Instrument using the LightCycler FastStart DNA Master SYBER Green I kit according to the manufacturer's instructions (Roche Applied Science). For each target gene, specific oligonucleotides were designed to amplify nucleotide fragments of 200-580 bp (see Supplementary Table S4, available with the online version of this paper, for oligonucleotide sequences). The amplification reactions were carried out with equal amounts of cDNA (120 ng), as initial template, and each reaction contained $0.5 \mu \mathrm{M}$ primers, $2 \mathrm{mM} \mathrm{MgCl}_{2}$, and hot-start PCR mix (Roche Applied Science). The expression ratio of the target gene was determined relative to a reference gene, the $E$. coli glyceraldehyde-3-phosphate dehydrogenase gene (gapA), which did not show variation in transcription abundance under any of the conditions tested by microarray analysis or real-time RT-PCR experiments.

Viability assays and complementation studies. In order to compare the sensitivity to CORM-2 of the E. coli parental strain versus the various mutant strains, cell viability was determined by evaluation of the number of colony-forming units (c.f.u. $\mathrm{ml}^{-1}$ ), after $7 \mathrm{~h}$ growth in the presence or in the absence of CORM-2. The percentage survival was calculated as the number of colonies obtained from CORM-2-treated cultures divided by the number of colonies formed upon plating untreated cultures. The experiments were performed in duplicate, with a minimum of two independent cultures of the mutant strain and of its corresponding parental strain (see Supplementary Table S5 for details of bacterial strains); the results are presented as means \pm SE. For complementation purposes, $i b p A B$ and $m e t R$ genes were amplified from $E$. coli genomic DNA using the appropriate oligonucleotides (Supplementary Table S4), and cloned into pUC19 to generate plasmids pibpAB and pmetR (see Supplementary Table S6 for details of plasmids). These plasmids or the empty vector were transformed into the correspondent mutant strain and the phenotype was analysed by plating on agar successive dilutions of the cultures treated with CORM-2 or left untreated. For $\Delta$ soxs, $\Delta o x y R, \Delta t s q A$ and $\Delta b h s A$, the complementation experiments were performed using plasmids pWB31, pAQ17, pCA24N $y d g G^{+}$and pCA24N $y c f R^{+}$, respectively (Supplementary Table S6).

Biofilm assays. Biofilm formation was quantified by the crystal violet method (Ren et al., 2005; Zhang et al., 2007). Briefly, overnight LB-grown cultures of wild-type E. coli, $\Delta t q s A$ and $\Delta b h s A$ mutant strains were used to inoculate fresh medium to an $\mathrm{OD}_{600}$ of 0.05 and then incubated for $6 \mathrm{~h}$, at $37^{\circ} \mathrm{C}$. At this stage, $250 \mu \mathrm{M}$ CORM- 2 was added and the plates were incubated for another $18 \mathrm{~h}$, at $37^{\circ} \mathrm{C}$. The total biofilm formed was stained with a solution of $0.1 \%$ crystal violet for $30 \mathrm{~min}$ and quantified by measuring the $A_{540}$. Relative biofilm formation was calculated as the total biofilm formed by the cultures treated with CORM-2 divided by the total biofilm obtained in untreated cultures; each data point represents the mean of six replicated wells from four independent cultures.

\section{RESULTS AND DISCUSSION}

\section{Analysis of the E. coli transcriptome of cells treated with CORM-2}

Microarray technology was utilized to analyse the genomewide transcriptional pattern of $E$. coli cells, grown aerobically and anaerobically, in the presence of CORM2. The complete list of genes that exhibited a twofold, or higher, increase or decrease (with $P<0.05$ ) in transcription is presented in Supplementary Tables S1 (anaerobic growth) and S2 (aerobic growth), available with the online version of this paper. In order to independently confirm the microarray results, quantitative real-time PCR was carried out on ten selected genes that were found to be upregulated or downregulated by the action of CORM-2. The values acquired in the real-time PCR experiments were in agreement with the fold variation obtained in the microarrays (Table 1). To facilitate subsequent analysis, the differentially expressed genes were divided into functional categories, based on the NCBI (http://www.ncbi.nlm.nih. gov) and EcoCyc (http://ecocyc.org) gene annotations and on the protein databases that were used to search for homologues of hypothetical gene products. Since a better correlation between gene expression and function is found for groups of genes instead of individual genes, the FatiGO algorithm (Al-Shahrour et al., 2004, 2006, 2007) was also used to interpret the data.

Anaerobically grown cells. The microarray data acquired for cells grown under anaerobic conditions and treated with CORM-2 showed that 396 genes had their transcription altered ( $\sim 9 \%$ of the total genome). Of these, the transcription of 228 genes was repressed $(\sim 5 \%$ of the transcriptome); these genes were dispersed through nearly all functional categories, particularly amino acid transport and metabolism (Fig. 1A). The FatiGO analysis showed that the genes downregulated are over-represented in cellular metabolic processes (which correspond to $84 \%$ of all repressed genes), namely in catabolic processes, nucleotide metabolism, and energy production by oxidation of organic compounds (Fig. 2A), indicating that CORM-2 induces metabolic adaptation.

The largest class of genes that were upregulated belongs to the category hypothetical proteins ( 39\%). Apart from these, the genes that showed increased transcription are divided essentially into three classes: inorganic ion transport, post-translational modification and transcription (Fig. 1A). Further analysis revealed that the genes upregulated by CORM-2 were significantly over-represented within the classes transcriptional regulation and protein folding (Fig. 2B). Among the latter, genes encoding two heat-shock proteins that are directly connected with 
Table 1. Quantitative real-time RT-PCR analysis performed in cells exposed to CORM-2 and to the $\mathrm{CO}$-free $\mathrm{Ru}(\mathrm{II}) \mathrm{Cl}_{2}(\mathrm{DMSO})_{4}$

\begin{tabular}{|c|c|c|c|c|c|c|}
\hline \multirow[t]{3}{*}{ Gene } & \multicolumn{3}{|c|}{ Anaerobic } & \multicolumn{3}{|c|}{ Aerobic } \\
\hline & \multirow[t]{2}{*}{ Microarray } & \multicolumn{2}{|c|}{ Real-time PCR $^{\star}$} & \multirow[t]{2}{*}{ Microarray } & \multicolumn{2}{|c|}{ Real-time PCR $^{\star}$} \\
\hline & & CORM-2 & $\mathrm{Ru}(\mathrm{II}) \mathrm{Cl}_{2}(\mathrm{DMSO})_{4}$ & & CORM-2 & $\mathrm{Ru}(\mathrm{II}) \mathrm{Cl}_{2}(\mathrm{DMSO})_{4}$ \\
\hline$i b p B$ & 40 & $427 \pm 33$ & $-2 \pm 0$ & 79 & $2798 \pm 27$ & $12 \pm 8$ \\
\hline $\operatorname{soxS}$ & 10 & $14 \pm 0$ & $-3 \pm 0$ & 15 & $268 \pm 63$ & $-3 \pm 0$ \\
\hline marA & 14 & $14 \pm 0$ & $2 \pm 1$ & 10 & $56 \pm 3$ & $-2 \pm 0$ \\
\hline frmA & 10 & $57 \pm 1$ & $-1 \pm 0$ & 16 & $133 \pm 0$ & $-2 \pm 0$ \\
\hline $\operatorname{lrh} A$ & 16 & $14 \pm 5$ & $-2 \pm 0$ & 5 & $23 \pm 8$ & $-2 \pm 0$ \\
\hline$y n c J$ & 42 & $85 \pm 8$ & $12 \pm 2$ & 62 & $162 \pm 49$ & $7 \pm 2$ \\
\hline$b h s A$ & 26 & $33 \pm 2$ & $3 \pm 2$ & - & - & - \\
\hline metR & - & - & - & 21 & $432 \pm 85$ & $4 \pm 0$ \\
\hline $\operatorname{gad} X$ & -10 & $-21 \pm 0$ & $2 \pm 1$ & -4 & $-4 \pm 0$ & $-2 \pm 0$ \\
\hline sucA & -9 & $-6 \pm 0$ & $1 \pm 0$ & -5 & $-4 \pm 0$ & $-1 \pm 0$ \\
\hline
\end{tabular}

${ }^{\star}$ Fold change \pm SE.

protein stability, $i b p A$ and $i b p B$, were strongly induced (19and 40-fold, respectively). The data also showed that, under anaerobic conditions, the addition of CORM-2 had effects on iron metabolism, since several genes related to this function were altered, e.g. those encoding ferritin $(\mathrm{ftn})$, and bacterioferritin ( $b f r)$ (Supplementary Table S1).

Aerobically grown cells. E. coli cells grown aerobically and exposed to CORM-2 displayed altered transcription of 175 genes, $\sim 4 \%$ of the global gene expression profile, with 46 genes repressed and 129 genes induced. Apart from genes encoding hypothetical proteins, three functional categories exhibited a similar number of transcriptionally repressed genes, namely the classes energy production, and amino acid and carbohydrate transport and metabolism (Fig. 1B). The FatiGO algorithm revealed over-representation of repressed genes in coenzyme catabolic processes, tricarboxylic acid cycle and aerobic respiration classes (Fig. 2C). This trend would ultimately lead to the inhibition of aerobic respiratory metabolism.

The genes whose expression was induced by CORM-2 were spread through several functional classes, the inorganic ion transport category containing the second-highest number of genes transcriptionally modified (Fig. 1B). Interestingly, the FatiGO analysis indicates that under aerobic conditions the addition of CORM-2 to E. coli cells led to a significant upregulation of genes involved in sulfur metabolism, such as tauABC, ssuAD, cysWA and $s b p$, and in methionine metabolism, like the gene clusters metNI and metBLF (Fig. 2D).

\section{Transcriptional alterations caused by CORM-2 are dependent on $\mathrm{CO}$ release}

Administration of any CORM produces some metabolite(s) along with the CO that is liberated. Therefore, we determined whether the biological effect of a given CORM actually results from the liberated $\mathrm{CO}$ and not from any of its metabolites or the CORM's molecular skeleton itself. The commercially available tricarbonyldichlororuthenium(II) dimer, $\left[\mathrm{Ru}(\mathrm{CO})_{3} \mathrm{Cl}_{2}\right]_{2}$, also known as CORM-2, is by far the most tested CORM and we have reported its bactericidal activity (Nobre et al., 2007). The dimer complex is insoluble in aqueous media and its solubilization, usually carried out in DMSO, entails a number of chemical transformations. According to Motterlini et al. (2002), $23 \mathrm{~min}$ after dissolution three species are already formed, namely $\mathrm{RuCl}_{2}(\mathrm{CO})_{3}(\mathrm{DMSO})$ and two isomers of $\mathrm{RuCl}_{2}(\mathrm{CO})_{2}$ (DMSO $)_{2}$. Of necessity, a certain amount of free $\mathrm{CO}$ is liberated into the DMSO solution and eventually lost to the atmosphere at an undetermined rate. More species appear upon standing or warming of the solution and further $\mathrm{CO}$ loss to give $\mathrm{RuCl}_{2}(\mathrm{CO})(\mathrm{DMSO})_{3}$, and eventually $\mathrm{RuCl}_{2}(\mathrm{DMSO})_{4}$ is to be expected since they are all synthetically interrelated in the presence of CO gas (Alessio et al., 1995). Nothing is presently known about the biological activity of any of these pure species individually. However, the octahedral structure, the oxidation state as well as the ligands other than $\mathrm{CO}$ are retained along this series, strongly supporting our choice of $\mathrm{Ru}(\mathrm{II}) \mathrm{Cl}_{2}(\mathrm{DMSO})_{4}$ as a control for the bactericidal activity of the CO-free $\mathrm{Ru}(\mathrm{II}) \mathrm{Cl}_{2}(\mathrm{DMSO})_{\mathrm{x}}$ fragment. It must also be mentioned that, regardless of the species present in the DMSO solution of $\left[\mathrm{Ru}(\mathrm{CO})_{3} \mathrm{Cl}_{2}\right]_{2}$, when an aliquot of this solution is added to the cell culture medium no $\mathrm{CO}$ is released to the gas phase, as previously reported (Nobre et al., 2007). We challenged $E$. coli with $500 \mu \mathrm{M}$ CO-free $\mathrm{Ru}(\mathrm{II}) \mathrm{Cl}_{2}(\mathrm{DMSO})_{4}$ and analysed the expression of several genes. Table 1 shows that no significant alteration in transcription was observed when the CO-free compound was used, which allows us to conclude that the main biological activity is due to the release of CO from CORM-2. 
A

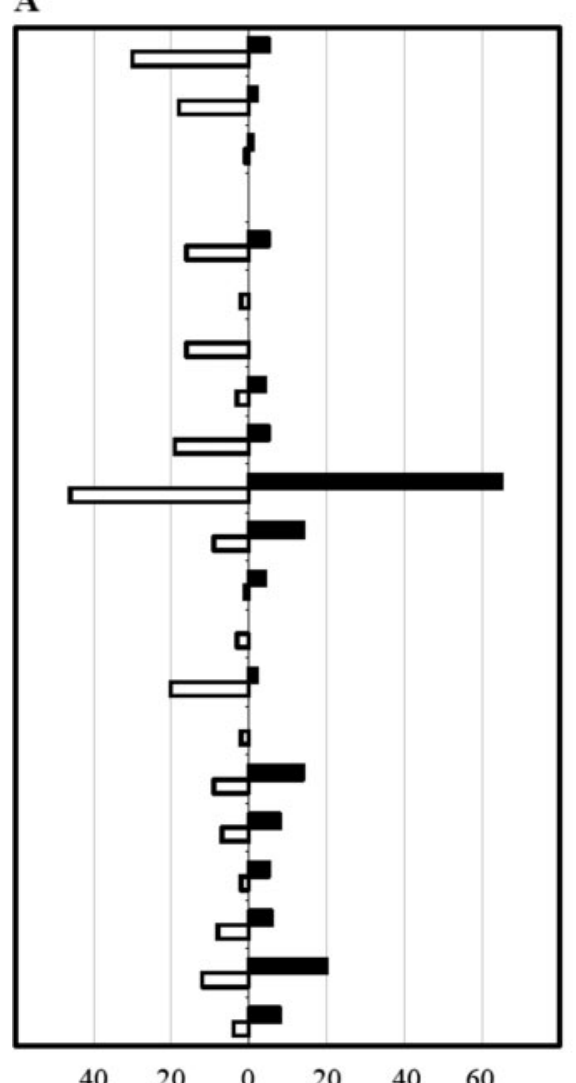

40
B

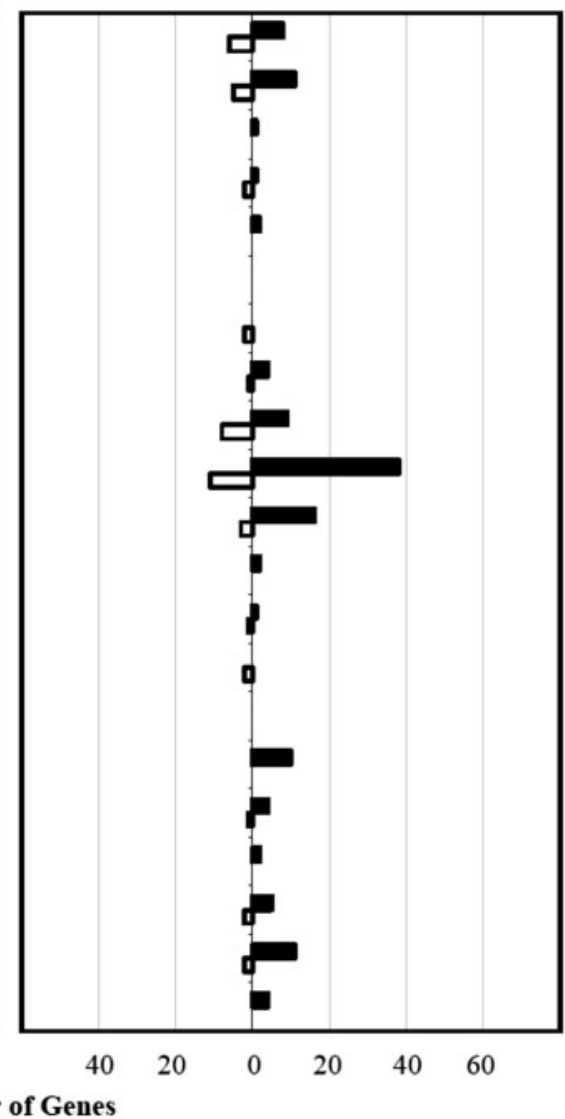

Amino Acid Transport and Metabolism

Carbohydrate Transport and Metabolism

Cell Cycle

Cell Motility

Cell Wall Biogenesis

Central Intermediary Metabolism

Coenzyme Transport and Metabolism

Defence Mechanisms

Energy Production and Conversion

Hypothetical Proteins

Inorganic Ion Transport and Metabolism

Intracellular Trafficking

Lipid Transport and Metabolism

Nucleotide Transport and Metabolism

Phage-Related Functions and Prophages

Posttranslational Modification and Chaperones

Replication, Recombination and Repair

Secondary Metabolites Biosynthesis, and Transport

Signal Transduction Mechanisms

Transcription

Translation, Ribosomal Structure and Biogenesis

Number of Genes
Functional Category

Fig. 1. Effect of CORM-2 on the E. coli transcriptome analysed according to the distribution of the genes with altered expression into functional categories. The differentially regulated genes in cells grown under anaerobic (A) and aerobic (B) conditions were divided into 21 functional categories according to the $\mathrm{NBCl}$ and EcoCyc databases. The black and white bars represent the number of genes whose transcription was up- and downregulated by CORM-2, respectively. The functional categories that include genes up- and downregulated by CORM-2 in both growth conditions are highlighted in bold, and the eight functional classes containing genes that were repressed by CORM-2 under anaerobic conditions and are absent in aerobically grown cells are shown in italics.

\section{Genes transcriptionally regulated in response to CORM-2, independently of oxygen}

The E. coli microarray data showed that upon exposure to CORM-2 the cells grown anaerobically exhibited more repressed genes than the cells grown aerobically. In particular, eight functional classes containing genes that were repressed by CORM-2 under anaerobic conditions were not detected in aerobically grown cells (Fig. 1A, B). These results are in agreement with our previous observation that the decrease in cell viability caused by CORMs is higher for cells grown under anaerobic conditions (Nobre et al., 2007). However, a large set of genes were found in common between the aerobic and anaerobic conditions, and the fold induction or repression generated by CORM-2 did not vary significantly between them (Table 2 ). There is also no example of a gene that was repressed under anoxic conditions and induced under aerobic conditions, and vice versa. The major fold difference was observed for the genes encoding a heat-shock protein $(i b p B)$, two hypothetical proteins $(y n c J$ and $y m g G)$, a flagellar repressor $(\operatorname{lrh} A)$ and an envelope-stress-induced periplasmic protein ( $s p y)$. In all these cases, the genes showed a higher fold CORM-2dependent induction in aerobically grown cells than in cells cultured anaerobically (Table 2 ).

\section{Phenotypic analysis of $E$. coli regulators induced by CORM-2}

To gain further insight into the function of the genes whose transcription was perturbed by treatment with CORM-2 we analysed the phenotype of $12 \mathrm{E}$. coli mutant strains to test if the deleted gene has a role in the E. coli CORM-2 response. The genes were chosen on the basis that they showed a high fold induction or repression and/or exhibited variation in transcript abundance in cells grown under both aerobic and anaerobic conditions (see Table 2). 
$\mathbf{A}$

C

Difference in percentage between the genes down-regulated and the reference genes
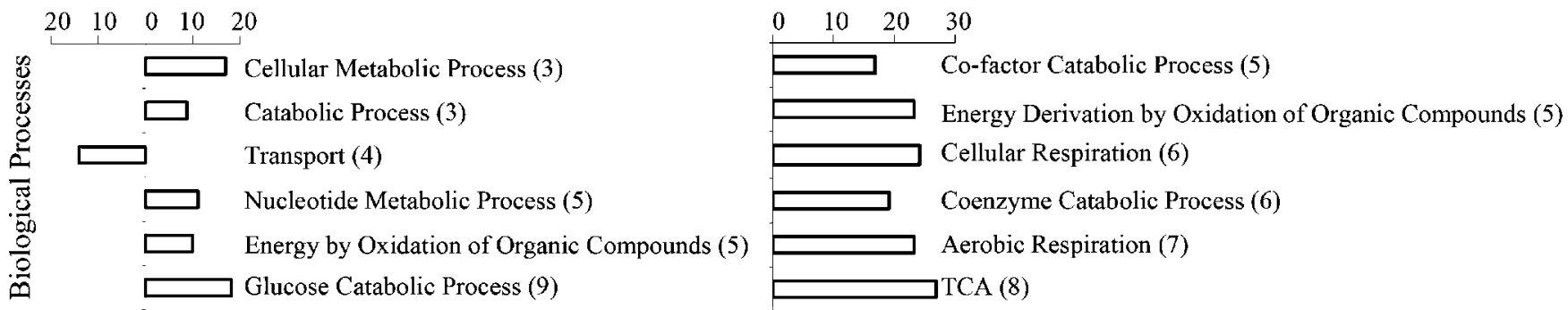

B

D

Difference in percentage between the genes up-regulated and the reference genes
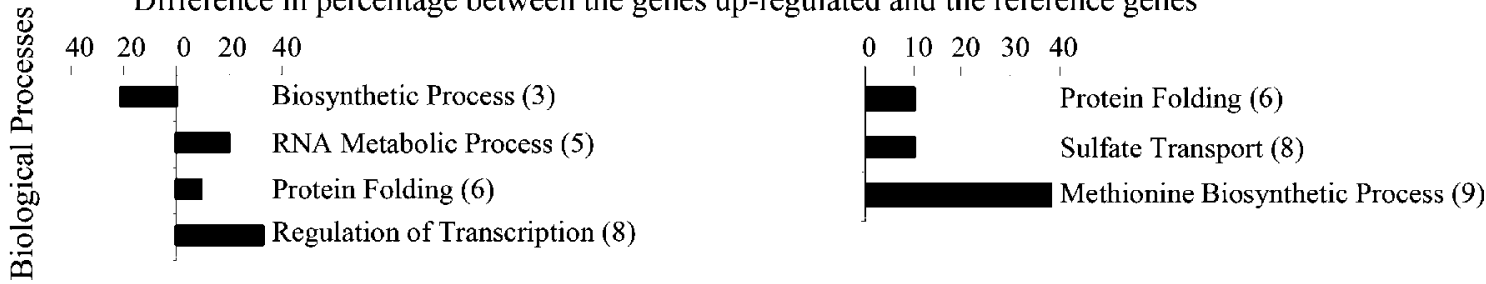

Fig. 2. Biological processes modified by CORM-2. The genes down- and upregulated by CORM-2 anaerobically (A and $B$ ) or aerobically ( $C$ and $D$ ) were compared with those unaltered by CORM-2 (reference genes), using the FatiGO algorithm (see Methods). The $y$-axis shows the biological processes that were considered differentially represented between the two groups compared in each case. The specificity of the biological process increases from the top to the bottom of the $y$-axis, as indicated by the numbers in parentheses. The $x$-axis represents the differences in percentages of the relative frequency of genes annotated for each biological process. The right and left part of the $x$-axis corresponds to the biological processes over- and under-represented, in the downregulated genes ( $A$ and $C$ ) and the upregulated genes (B and $D$ ), respectively. TCA, tricarboxylic acid cycle.

Hence, genes encoding regulators (soxS, oxyR, zntR, metR, $\operatorname{gad} X)$, a putative stress-combating protein $(\operatorname{cpxP} P)$, heatshock proteins ( $i b p A$ and $i b p B)$, proteins involved in biofilm formation $[y d g G(t q s A), y c f R(b h s A)]$, an antiporter protein (chaA), and genes involved in methionine metabolism (metN, metI) were studied.

Study of the E. coli single mutant strains deleted in the genes zntR, gadX and chaA revealed that these strains did not display a CORM-2 growth-induced delay when compared to the parent strain (data not shown).

The soxS gene, whose transcription is controlled directly by SoxR, showed a high fold induction upon treatment with CORM-2, under both aerobic and anaerobic conditions (Table 2). Together with SoxR, SoxS participates in the regulation of several genes involved in the response to oxidative stress (Wu \& Weiss, 1992). However, with the exception of the marRAB operon, none of the other known members of the SoxRS regulon was induced. A similar behaviour, which so far remains unexplained, was previously observed in other microarray experiments with E. coli grown in the presence of nitric oxide and hydrogen peroxide (Justino et al., 2005; Zheng et al., 2001). The phenotypic study of the E. coli strain deficient in the soxS gene showed that the mutation leads to an increased sensitivity to CORM-2 of the cells grown under aerobic $(>70 \%)$ and anaerobic conditions (>35\%) (Fig. 3A, B).

The $\operatorname{oxy} R$ gene, whose encoded protein participates in the regulation of genes involved in the response to oxidative stress (Zheng et al., 2001), was upregulated by CORM-2 under aerobic and anaerobic conditions ( $\geqslant 4$-fold) (Table 2). Under aerobic conditions and in the presence of CORM-2, the sensitivity of the $\operatorname{oxy} R$ mutant strain was found to be $\sim 70 \%$ higher than that of the wild-type strain (Fig. 3C), while no differences were observed for growth under anaerobic conditions.

The microarray data showed that in aerobically grown $E$. coli the transcription of $m e t R$ was upregulated by CORM- 2 . Analysis of the $\Delta m e t R$ strain revealed that this mutant displays a sensitivity $\sim 80 \%$ higher than that of the parental strain for cells grown aerobically (Fig. 3F), while no effect was detected in anoxic conditions (data not shown).

In all cases, the wild-type behaviour was restored upon expression of the genes from a plasmid containing SoxR, OxyR or MetR (Fig. $3 A_{1}-C_{1}$ and $F_{1}$ ), showing that these transcription factors have an important role in the E. coli regulatory mechanisms triggered by exposure to CORM-2. 
Table 2. Genes of E. coli differentially regulated by CORM-2 under both anaerobic and aerobic conditions

\begin{tabular}{|c|c|c|c|c|}
\hline \multirow[t]{2}{*}{ Gene name ${ }^{\star}$} & \multirow[t]{2}{*}{ Locus } & \multirow[t]{2}{*}{ Gene product } & \multicolumn{2}{|c|}{ Fold change } \\
\hline & & & Anaerobic & Aerobic \\
\hline alx & b3088 & Predicted inner-membrane protein & 12.3 & 3.3 \\
\hline yheL & b3343 & Predicted intracellular sulfur oxidation protein & 6.8 & 7.4 \\
\hline exuT & b3093 & Hexuronate transporter & 6.5 & 2.5 \\
\hline $\operatorname{met} N$ & b0199 & D-Methionine transport ATP-binding protein & 4.3 & 9.9 \\
\hline metI & b0198 & D-Methionine transport system permease & 4.0 & 6.5 \\
\hline$p s p E$ & b1308 & Phage shock protein E precursor & 2.4 & 7.0 \\
\hline yegH & b2063 & Putative transport protein & -2.4 & -2.5 \\
\hline$a p a G$ & b0050 & Uncharacterized protein associated with $\mathrm{Co}^{2+} / \mathrm{Mg}^{2+}$ efflux & -3.6 & -2.4 \\
\hline soxs & b4062 & DNA-binding transcriptional dual regulator & 10.0 & 14.5 \\
\hline frmR & b0357 & Regulator protein that represses frm $R A B$ operon & 8.5 & 6.7 \\
\hline marA & b1531 & Multiple antibiotic-resistance protein & 7.3 & 9.5 \\
\hline $\operatorname{lex} A$ & b4043 & LexA repressor & 6.8 & 3.8 \\
\hline$z n t R$ & b3292 & Zinc-responsive transcriptional regulator & 5.1 & 6.1 \\
\hline oxyR & b3961 & Hydrogen peroxide-inducible genes activator & 4.5 & 4.1 \\
\hline $\operatorname{gadX}$ & b3516 & DNA-binding transcriptional dual regulator & -10.4 & -4.3 \\
\hline \multicolumn{5}{|c|}{ Post-translational modification, protein turnover, chaperones } \\
\hline$i b p B$ & b3686 & $16 \mathrm{kDa}$ heat-shock protein $\mathrm{B}$ & 39.6 & 79.3 \\
\hline$i b p A$ & b3687 & $16 \mathrm{kDa}$ heat-shock protein $\mathrm{A}$ & 19.2 & 18.7 \\
\hline$h s p Q$ & b0966 & DNA-binding protein, hemimethylated & 7.7 & 4.8 \\
\hline yeeD & b2012 & Predicted redox protein & 7.4 & 3.3 \\
\hline$p s p G$ & b4050 & Phage shock protein $\mathrm{G}$ & 3.9 & 11.4 \\
\hline narP & b2193 & Nitrate/nitrite response regulator protein & 2.7 & 2.8 \\
\hline$y d i V$ & b1707 & Cyclic-di-GMP phosphodiesterase class I & -3.7 & -5.9 \\
\hline \multicolumn{5}{|c|}{ Energy production and conversion } \\
\hline frmA & b0356 & Alcohol dehydrogenase class III & 15.8 & 8.1 \\
\hline nemA & b1650 & $N$-Ethylmaleimide reductase & 3.1 & 3.6 \\
\hline$s u c A$ & b0726 & 2-Oxoglutarate decarboxylase & -9.1 & -5.1 \\
\hline \multicolumn{5}{|c|}{ Translation, ribosomal structure and biogenesis } \\
\hline rrmJ & b3179 & rRNA large subunit methyltransferase J & 4.6 & 3.2 \\
\hline$h s l R$ & b3400 & Ribosome-associated heat-shock protein Hsp15 & 4.2 & 5.1 \\
\hline miaA & b4171 & tRNA $\Delta^{2}$-isopentenylpyrophosphate transferase & 2.5 & 2.5 \\
\hline \multicolumn{5}{|c|}{ Amino acid transport and metabolism } \\
\hline$y b a T$ & b0486 & Predicted transporter protein & -3.4 & -3.7 \\
\hline$g s s$ & b2988 & Fused glutathionylspermidine amidase & -3.5 & -3.1 \\
\hline \multicolumn{5}{|c|}{ Carbohydrate transport and metabolism } \\
\hline$t k t B$ & b2465 & Transketolase & -2.5 & -2.9 \\
\hline ots $A$ & b1896 & $\alpha, \alpha$-Trehalose-phosphate synthase & -4.9 & -2.8 \\
\hline \multicolumn{5}{|c|}{ Replication, recombination and repair } \\
\hline fimE & b4313 & Tyrosine recombinase & 5.6 & 3.9 \\
\hline $\operatorname{rec} N$ & b2616 & Recombination and repair protein & 5.4 & 8.4 \\
\hline
\end{tabular}


Table 2. cont.

\begin{tabular}{|c|c|c|c|c|}
\hline \multirow[t]{2}{*}{ Gene name ${ }^{\star}$} & \multirow[t]{2}{*}{ Locus } & \multirow[t]{2}{*}{ Gene product } & \multicolumn{2}{|c|}{ Fold change } \\
\hline & & & Anaerobic & Aerobic \\
\hline \multicolumn{5}{|c|}{ Defence mechanisms } \\
\hline$r d o A$ & b3859 & Thr/Ser kinase implicated in Cpx stress response & 2.8 & 3.5 \\
\hline aidB & b4187 & Isovaleryl-CoA dehydrogenase & -2.6 & -2.7 \\
\hline \multicolumn{5}{|c|}{ Coenzyme transport and metabolism } \\
\hline$b t u D$ & b1709 & Vitamin B12-transporter ATPase & -3.1 & -2.3 \\
\hline \multicolumn{5}{|c|}{ Cell wall/membrane/envelope biogenesis } \\
\hline amiC & b2817 & $N$-Acetylmuramoyl-L-alanine amidase & 2.4 & 2.4 \\
\hline \multicolumn{5}{|c|}{ Secondary metabolites biosynthesis, transport and catabolism } \\
\hline$y b b A$ & b0495 & Hypothetical ABC transporter & 7.7 & 7.1 \\
\hline \multicolumn{5}{|c|}{ Intracellular trafficking, secretion, and vesicular transport } \\
\hline spy & b1743 & Envelope-stress-induced periplasmic protein & 16.0 & 30.0 \\
\hline \multicolumn{5}{|c|}{ Cell cycle control, cell division, chromosome partitioning } \\
\hline sulA & b0958 & Cell division inhibitor & 9.5 & 5.4 \\
\hline \multicolumn{5}{|c|}{ Hypothetical proteins } \\
\hline$y n c J$ & b1436 & Hypothetical protein & 42.2 & 62.0 \\
\hline yjfN & b4188 & Hypothetical protein & 19.8 & 22.9 \\
\hline$y c f J$ & b1110 & Hypothetical protein & 18.0 & 13.5 \\
\hline yebE & b1846 & Hypothetical protein & 16.3 & 22.9 \\
\hline$y c f S$ & b1113 & Hypothetical protein & 12.0 & 16.9 \\
\hline$y h d V$ & b3267 & Hypothetical protein & 9.0 & 9.0 \\
\hline yneM & b4599 & Hypothetical protein & 8.4 & 6.2 \\
\hline yqjA & b3095 & Hypothetical protein & 8.0 & 5.7 \\
\hline$y p f G$ & b2466 & Hypothetical protein & 6.7 & 8.6 \\
\hline$f x s A$ & b4140 & FxsA protein & 6.7 & 13.8 \\
\hline yaiY & b0379 & Hypothetical protein & 6.5 & 5.8 \\
\hline $\operatorname{tqs} A$ & b1601 & Predicted permease & 6.3 & 9.1 \\
\hline yeeE & b2013 & Predicted inner-membrane protein & 6.3 & 2.7 \\
\hline$y b e D$ & b0631 & Hypothetical protein & 6.1 & 6.0 \\
\hline yqaE & b2666 & Hypothetical protein & 5.9 & 4.5 \\
\hline$y t f K$ & b4217 & Hypothetical protein & 5.3 & 5.3 \\
\hline$y c j X$ & b1321 & Hypothetical protein & 5.0 & 7.7 \\
\hline$y b f A$ & b0699 & Hypothetical protein & 4.5 & 4.5 \\
\hline bax & b3570 & BAX protein & 4.3 & 3.3 \\
\hline$y m g G$ & b1172 & Hypothetical protein & 4.2 & 16.0 \\
\hline sraF & b4448 & Unknown RNA & 3.5 & 2.5 \\
\hline$y r f G$ & b3399 & Predicted hydrolase & 3.4 & 4.3 \\
\hline$y n f D$ & b1586 & Hypothetical protein & 3.4 & 4.5 \\
\hline$y c i S$ & b1279 & Hypothetical protein & 3.3 & 3.2 \\
\hline$y c j F$ & b1322 & Hypothetical protein & 3.2 & 10.0 \\
\hline$y h d N$ & b3293 & Hypothetical protein & 3.1 & 4.3 \\
\hline$y c i C$ & b1255 & Hypothetical protein & 2.6 & 2.3 \\
\hline$s l p$ & b3506 & Outer-membrane protein Slp precursor & -2.2 & -4.1 \\
\hline yahK & b0325 & Predicted oxidoreductase, Zn-dependent and NAD(P)-binding & -4.0 & -3.6 \\
\hline$y c i X$ & b4523 & Hypothetical protein & -4.6 & -8.0 \\
\hline
\end{tabular}

${ }^{\star}$ Strains mutated in the genes shown in bold were analysed phenotypically.

\section{Identification of other genes involved in the CORM-2 sensitivity of anaerobically grown $E$. coli cells}

A significant variation of the expression of $i b p A$ and $i b p B$ genes was measured in aerobically and anaerobically grown cells of E. coli treated with CORM-2 (Table 2). These genes encode two small heat-shock proteins, IbpA and IbpB, that bind to protein aggregates and inclusion bodies formed during heterologous protein expression (Lethanh et al., 2005). The two proteins are known to cooperate with $\mathrm{ClpB}$ and DnaK, forming a functional triad of chaperones (Lethanh et al., 2005; Thomas \& Baneyx, 1998). Interestingly, the transcription of $c l p B$ and $d n a K$ as well as that of 

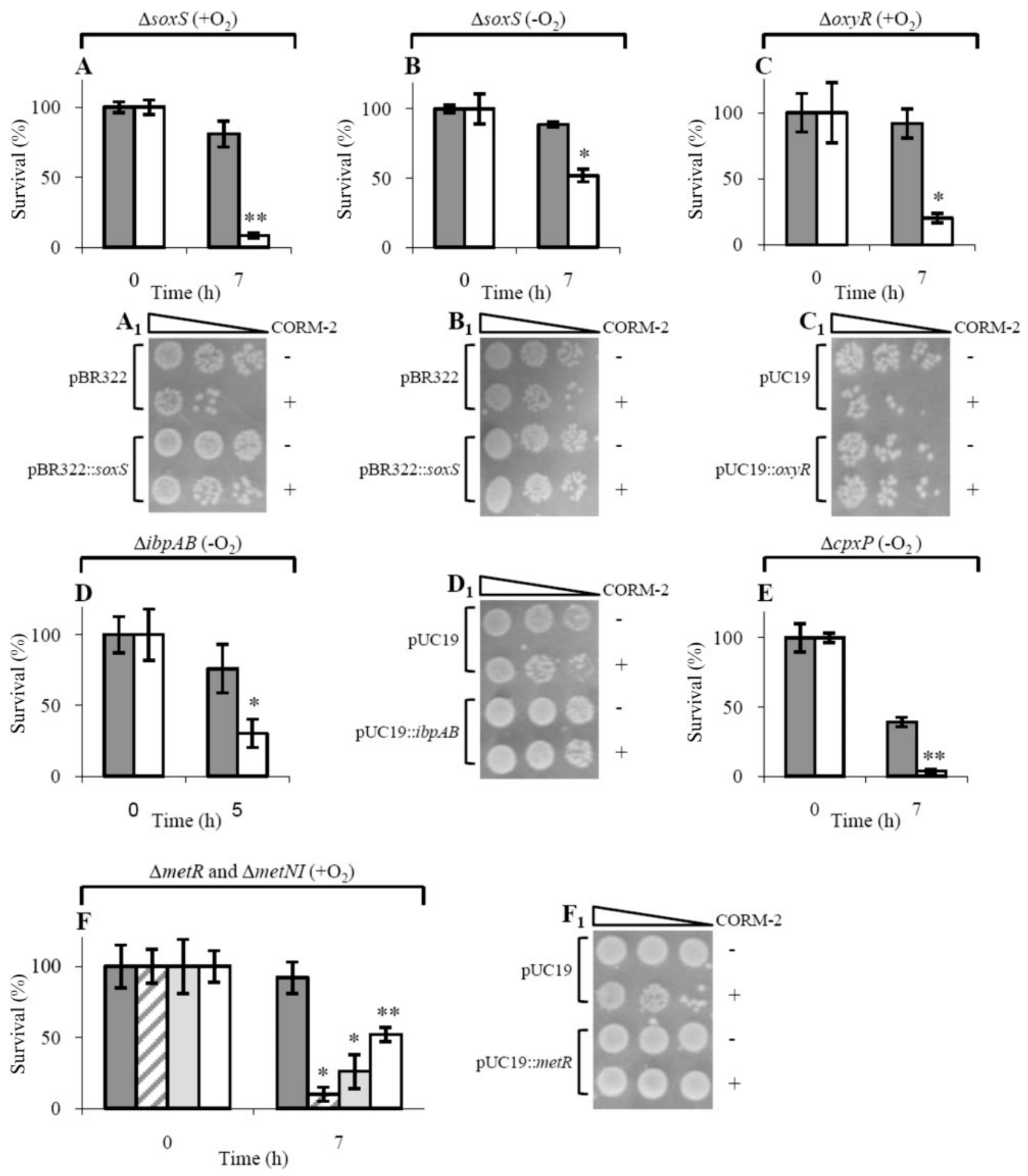

Fig. 3. Analysis of the sensitivity of various $E$. coli mutant strains to CORM-2. (A-E) The parental strain (grey bars), and mutants (white bars) were grown aerobically (panels $A$ and $C$ ) or anaerobically (panels $B, D$ and $E$ ) and exposed to $150 \mu \mathrm{M}$ or $100 \mu \mathrm{M}$ CORM-2, respectively. The E. coli $\Delta c p x P$ mutant and its parental strain were treated with $125 \mu \mathrm{M}$ CORM-2 (panel E). In panel $\mathrm{F}$, the $\Delta$ metR (striped bars), $\Delta$ metl (light grey bars) and $\Delta$ met $N$ (white bars) mutant strains were grown aerobically and treated with $150 \mu \mathrm{M}$ CORM-2. The error bars represent the standard error of the mean values obtained from at least two independent biological samples performed in duplicate $\left({ }^{\star} P<0.01,{ }^{\star *} P<0.05\right)$. Panels $A_{1}-F_{1}$ : complementation analysis of $\operatorname{sox} S\left(\mathrm{~A}_{1}, \mathrm{~B}_{1}\right), \operatorname{\Delta oxy} R\left(\mathrm{C}_{1}\right), \Delta i b p A B\left(\mathrm{D}_{1}\right)$ and $\Delta$ metR $\left(\mathrm{F}_{1}\right)$ using the plasmids indicated in each panel. 
genes coding for other chaperones, such as dnaJ, grpE and $h t p G$, was also upregulated anaerobically by CORM-2 (Supplementary Table S1). Under anaerobic conditions, inactivation of the $i b p A B$ genes led to a $45 \%$ increase in CORM-2 sensitivity relative to the parental strain (Fig. 3D), while no differences were observed under aerobic conditions (data not shown). As expected, complementation of $i b p A B$ mutation induced the rescue of the wild-type phenotype (Fig. $3 \mathrm{D}_{1}$ ).

The transcription of E. coli $c p x P$, which encodes a periplasmic protein putatively involved in combating extracytoplasmic protein-mediated toxicity (Danese \& Silhavy, 1998), was upregulated 12-14-fold in cells exposed to CORM-2 and cultured in both aerobic and anaerobic conditions. CpxP belongs to the $E$. coli $\mathrm{Cpx}$ system, which senses perturbations in the bacterial cell envelope and responds through the upregulation of many gene products involved in protein folding and degradation (Fleischer et al., 2007). Accordingly, we verified that together with the induction of $c p x P$ occurred a high number of transcriptionally modified genes involved in cell wall biogenesis and in protein folding, including genes encoding heat-shock proteins ( $i b p A B, h s l J, h t p X)$, chaperones $(d n a J, h t p G, c l p B)$ and proteases $(f t s H)$ (Supplementary Table S1), mainly under anaerobic conditions. These genes are under the control of $r p o H\left(\sigma^{32}\right)$ (Zhao et al., 2005), a gene that was also found to be upregulated by CORM-2 under anaerobic conditions. The study of the growth behaviour of the $\Delta c p x P$ mutant revealed that this strain is approximately $35 \%$ more sensitive to CORM-2 than the parental strain, but only under anaerobic conditions (Fig. 3E), not under aerobic conditions (data not shown).

\section{CORM-2 interferes with methionine biosynthesis}

Addition of CORM-2 to aerobically grown cells of E. coli caused a marked increase in the transcription of several genes implicated in methionine biosynthesis and uptake, with $m e t R$ being the gene that showed the highest induction (21-fold) (Supplementary Table S2). Apart from the genes listed in Supplementary Table S2, induction of the expression of metA (12.4-fold; $P=0.0527)$ and metE (5.5-fold; $P=0.0565)$ was also observed. To further clarify this issue, growth experiments were conducted aerobically in the presence of CORM-2 for the E. coli $\Delta m e t R, \Delta m e t I$ and $\Delta m e t N$ mutant strains. All these mutants were hypersensitive to CORM-2 (80\%, 66\% and $40 \%$ higher sensitivity, respectively; Fig. 3F). Furthermore, the complementation assay showed that E. coli $\Delta m e t R$ regains resistance to $\mathrm{CO}$ upon expression of the regulator MetR (Fig. $3 \mathrm{~F}_{1}$ ). These findings suggest that, under aerobic conditions, CORM-2 affects the metabolism of methionine by E. coli.

\section{CORM-2 influences biofilm formation}

Previous microarray studies revealed that apart from the E. coli genes that are directly implicated in the process of biofilm formation, such as tqsA, mqsR, bhsA, yceP (bssS) and $y l i H(b s s)$, genes involved in the response to stress conditions are also induced during biofilm formation, namely ibpAB, soxS, $c p x P$ and spy (Beloin et al., 2004; Ren et al., 2004). Many of these genes were also found to be transcriptionally modified when $E$. coli is exposed to CORM-2 (Tables 2 and S3). In particular, comparison of the microarray data showed that approximately $40 \%$ of the genes that are transcriptionally modified during the formation of E. coli biofilm (Ren et al., 2004) are in common with those altered by CORM-2 under anaerobic conditions, and $12 \%$ of the common genes are observed when growth is performed aerobically (Supplementary Table S3).

To evaluate the effect of CORM-2 in the process of biofilm formation, quantification of the total biofilm formed during aerobic growth in LB and in the presence of the compound was performed. The results showed that CORM-2 increased by 1.6-fold the total biofilm of the $E$. coli wild-type strain (Fig. 4A). This indicates that E. coli produces more biofilm as a defensive response against CORM-2, as previously observed for other stress conditions (Zhang et al., 2007).

The $b s h A$ gene, which is related to biofilm formation (Zhang et al., 2007), was significantly induced under anaerobic conditions (26-fold). Although the $\Delta b h s A$ mutant showed an elevated resistance to CORM-2 anaerobically (Fig. 4B), and the complementation experiment performed with a multiple-copy clone containing the bhsA regulatory region rescued the wild-type behaviour (Fig. $4 \mathrm{~B}_{2}$ ), the values of total biofilm measured for the mutant strain exposed to CORM-2 matched those of the parental strain (Fig. 4A).

In E. coli, the tqsA gene is proposed to encode a putative transport protein involved in biofilm formation (Herzberg et al., 2006). This gene was upregulated in aerobically and anaerobically grown CORM-2-treated cells, with a 9- and 6-fold increase, respectively. Analysis of the E. coli $\Delta t q s A$ mutant revealed that deletion of $t q s A$ yielded a strain with higher resistance to CORM-2 than the parental strain, under both aerobic and anaerobic growth conditions (Fig. $4 \mathrm{~B}, \mathrm{C}$ ), and the phenotype of the tqs $A$ mutant strain could be fully complemented by a clone containing the tqsA gene (Fig. $4 \mathrm{~B}_{1}-\mathrm{C}_{1}$ ). This result is in agreement with those obtained by Herzberg et al. (2006), which showed that inactivation of tqsA increases the resistance to several antibiotics. Additionally, deletion of tqs $A$ abolished the increase in biofilm formation observed in the wild-type strain upon exposure to CORM-2 (Fig. 4A).

\section{Conclusion}

In this study, we provide the first microarray analysis of a micro-organism treated with the new bactericide CORM-2. The choice of $E$. coli was based on the fact that, besides being a model bacterium, this Gram-negative microorganism is able to live in aerobic environments and also adapts to anaerobic niches as part of its normal 

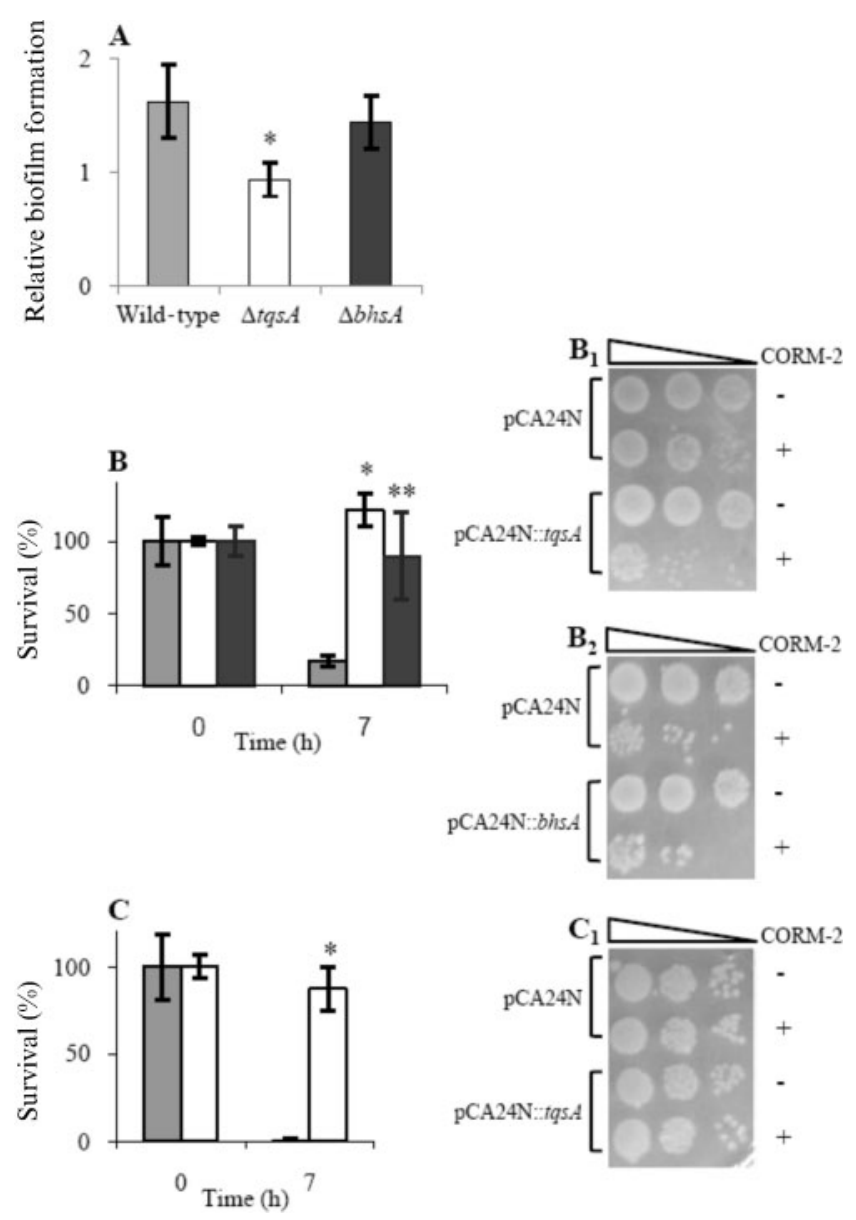

Fig. 4. Effect of CORM-2 on biofilm formation and cell viability of E. coli $\Delta t q s A$ and $\Delta b h s A$ mutant strains. (A) Biofilm was assayed in E. coli wild-type and in $\Delta t q s A$ and $\Delta b h s A$ mutant strains, in the absence or presence of $250 \mu \mathrm{M}$ CORM-2. (B, C) Cell viability of E. coli parental strain (grey bars), $\Delta t q s A$ (white bars) and $\Delta b h s A$ (black bars) mutants grown anaerobically (b) or aerobically (c) in the presence of $100 \mu \mathrm{M}$ or $180 \mu \mathrm{M}$ CORM-2, respectively. The number of c.f.u. was determined for at least two biological samples and in duplicate. Error bars represent the standard error of mean values $\left({ }^{\star} P<0.01,{ }^{\star \star} P<0.05\right)$. Complementation analysis of $\Delta t q s A$ and $\Delta b h s A\left(\mathrm{~B}_{1}\right.$ and $\mathrm{B}_{2}$, anaerobic conditions) and $\Delta t q s A\left(\mathrm{C}_{1}\right.$, aerobic conditions) mutant strains, using the plasmids indicated in each panel, is also shown.

colonization-transmission cycle within a host. Hence, it allowed us to compare the transcriptional response of a bacterium when treated with CORM-2 in both anaerobic and aerobic environments. The broad effect of CORM-2 on gene expression levels is evident from the distribution of induced and repressed genes over the spectrum of all functional categories. In particular, the higher number of genes affected in anaerobically CORM-2-exposed cells illustrates fundamental differences between the way in which microbes control external stress under the two oxygen conditions, and highlights the importance of specific investigations to understand the different adaptation strategies.

Although it has been assumed that the targets of $\mathrm{CO}$ are haem-containing enzymes or proteins, they have not yet been identified and little is known about the trigger event or mechanism that mediates the transfer of $\mathrm{CO}$ from the CORM to the haem target. The present data show that the action of $\mathrm{CO}$ extends beyond the action on haem proteins since a wide range of transcriptional modifications is observed in cells grown under fermentative conditions and exposed, aerobically or anaerobically, to CORM-2.

The changes in the expression level of key transcription regulators together with the phenotypic analysis of the mutant strains reveal that CORM-2 triggers a complex network of responses. As observed in all other microarray studies performed in E. coli cells submitted to toxic chemicals such as hydrogen peroxide and NO, exposure to CORM-2 increases the transcription of the soxS regulator but it does not induce any of the genes of the regulon, a result that requires further studies for clarification. A particular observation, common to all transcriptional studies, is the large representation of 'unknown' genes, which leaves a wide field to be still explored, until bacterial physiology is fully understood and indeed ready for the application of a systems biology approach. Nevertheless, the data provided by this study will certainly be valuable for guiding future research on the pharmacological application of CORMs.

\section{ACKNOWLEDGEMENTS}

The authors are grateful to Professor Bernard Weiss for providing E. coli $\Delta$ soxS, E. coli BW748 and BW1068, Professor François Baneyx for E. coli $\triangle i b p A B$, Professor Thomas Silhavy and Dr Dan Isaac for the $\Delta c p x P$ mutant, Professor Robert Poole and Professor Frederick Blattner for the $E$. coli strains mutated in methionine-related genes $(\Delta m e t R, \Delta m e t I$ and $\Delta m e t N)$, Dr Kobayashi Hiroshi for the $\Delta c h a A$ mutant, Professor John Foster for the $\Delta g a d X$ mutant, Professor Robert Poole and Dr Andrew Morby for the $\Delta z n t R$ mutant, Professor Thomas Wood and Dr Rodolfo Contreras for the $\Delta t q s A$ and $\Delta b h s A$ mutants and E. coli AG1 containing the pCA24N, pCA24NtqsA ${ }^{+}$and pCA24NbhsA ${ }^{+}$vectors, and Dr Gisela Storz for the pAQ17 vector. We thank Professor Miguel Teixeira and Professor Carlos Romão from ITQB for helpful discussions. This work was supported by FCT project POCI/SAU-IMI/56088/2004 and L.S. N. is recipient of $\mathrm{PhD}$ grant FCT SFRH/BD/22425/2005.

\section{REFERENCES}

Alessio, E., Milani, B., Bolle, M., Mestroni, G., Faleschini, P., Todone, F., Geremia, S. \& Calligaris, M. (1995). Carbonyl derivatives of chloride-dimethyl sulfoxide-ruthenium(II) complexes: synthesis, structural characterization, and reactivity of $\mathrm{Ru}(\mathrm{CO})_{\mathrm{x}}(\mathrm{DMSO})_{4}-x \mathrm{Cl}_{2}$ complexes $(x=1-3)$. Inorg Chem 34, 4722-4734.

Al-Shahrour, F., Diaz-Uriarte, R. \& Dopazo, J. (2004). FatiGO: a web tool for finding significant associations of Gene Ontology terms with groups of genes. Bioinformatics 20, 578-580.

Al-Shahrour, F., Minguez, P., Tarraga, J., Montaner, D., Alloza, E., Vaquerizas, J. M., Conde, L., Blaschke, C., Vera, J. \& Dopazo, J. (2006). BABELOMICS: a systems biology perspective in the 
functional annotation of genome-scale experiments. Nucleic Acids Res 34, W472-W476.

Al-Shahrour, F., Minguez, P., Tarraga, J., Medina, I., Alloza, E., Montaner, D. \& Dopazo, J. (2007). FatiGO +: a functional profiling tool for genomic data. Integration of functional annotation, regulatory motifs and interaction data with microarray experiments. Nucleic Acids Res 35, W91-W96.

Beloin, C., Valle, J., Latour-Lambert, P., Faure, P., Kzreminski, M., Balestrino, D., Haagensen, J. A., Molin, S., Prensier, G. \& other authors (2004). Global impact of mature biofilm lifestyle on Escherichia coli K-12 gene expression. Mol Microbiol 51, 659-674.

Danese, P. N. \& Silhavy, T. J. (1998). CpxP, a stress-combative member of the Cpx regulon. J Bacteriol 180, 831-839.

Dulak, J., Jozkowicz, A., Foresti, R., Kasza, A., Frick, M., Huk, I., Green, C. J., Pachinger, O., Weidinger, F. \& Motterlini, R. (2002). Heme oxygenase activity modulates vascular endothelial growth factor synthesis in vascular smooth muscle cells. Antioxid Redox Signal 4, 229-240.

Fleischer, R., Heermann, R., Jung, K. \& Hunke, S. (2007). Purification, reconstitution, and characterization of the CpxRAP envelope stress system of Escherichia coli. J Biol Chem 282, 8583-8593.

Herzberg, M., Kaye, I. K., Peti, W. \& Wood, T. K. (2006). YdgG (TqsA) controls biofilm formation in Escherichia coli K-12 through autoinducer 2 transport. J Bacteriol 188, 587-598.

Justino, M. C., Vicente, J. B., Teixeira, M. \& Saraiva, L. M. (2005). New genes implicated in the protection of anaerobically grown Escherichia coli against nitric oxide. J Biol Chem 280, 2636-2643.

Lethanh, H., Neubauer, P. \& Hoffmann, F. (2005). The small heatshock proteins IbpA and IbpB reduce the stress load of recombinant Escherichia coli and delay degradation of inclusion bodies. Microb Cell Fact 4, 6 .

Li, C. \& Hung Wong, W. (2001). Model-based analysis of oligonucleotide arrays: model validation, design issues and standard error application. Genome Biol 2, RESEARCH0032.

Li, C. \& Wong, W. H. (2001). Model-based analysis of oligonucleotide arrays: expression index computation and outlier detection. Proc Natl Acad Sci U S A 98, 31-36.

Li, C., Hossieny, P., Wu, B. J., Qawasmeh, A., Beck, K. \& Stocker, R. (2007). Pharmacologic induction of heme oxygenase-1. Antioxid Redox Signal 9, 2227-2239.

Motterlini, R., Clark, J. E., Foresti, R., Sarathchandra, P., Mann, B. E. \& Green, C. J. (2002). Carbon monoxide-releasing molecules: characterization of biochemical and vascular activities. Circ Res 90, E17-E24.

Motterlini, R., Mann, B. E. \& Foresti, R. (2005). Therapeutic applications of carbon monoxide-releasing molecules. Expert Opin Investig Drugs 14, 1305-1318.
Nobre, L. S., Seixas, J. D., Romao, C. C. \& Saraiva, L. M. (2007). Antimicrobial action of carbon monoxide-releasing compounds. Antimicrob Agents Chemother 51, 4303-4307.

Otterbein, L. E. \& Choi, A. M. (2000). Heme oxygenase: colors of defense against cellular stress. Am J Physiol Lung Cell Mol Physiol 279, L1029-L1037.

Ren, D., Bedzyk, L. A., Thomas, S. M., Ye, R. W. \& Wood, T. K. (2004). Gene expression in Escherichia coli biofilms. Appl Microbiol Biotechnol 64, 515-524.

Ren, D., Zuo, R., Gonzalez Barrios, A. F., Bedzyk, L. A., Eldridge, G. R., Pasmore, M. E. \& Wood, T. K. (2005). Differential gene expression for investigation of Escherichia coli biofilm inhibition by plant extract ursolic acid. Appl Environ Microbiol 71, 4022-4034.

Ryter, S. W., Alam, J. \& Choi, A. M. (2006). Heme oxygenase-1/carbon monoxide: from basic science to therapeutic applications. Physiol Rev 86, 583-650.

Stupfel, M. \& Bouley, G. (1970). Physiological and biochemical effects on rats and mice exposed to small concentrations of carbon monoxide for long periods. Ann N Y Acad Sci 174, 342-368.

Thomas, J. G. \& Baneyx, F. (1998). Roles of the Escherichia coli small heat shock proteins IbpA and IbpB in thermal stress management: comparison with $\mathrm{ClpA}, \mathrm{ClpB}$, and HtpG in vivo. J Bacteriol 180, 5165-5172.

Verma, A., Hirsch, D. J., Glatt, C. E., Ronnett, G. V. \& Snyder, S. H. (1993). Carbon monoxide: a putative neural messenger. Science 259, 381-384.

Wu, L. \& Wang, R. (2005). Carbon monoxide: endogenous production, physiological functions, and pharmacological applications. Pharmacol Rev 57, 585-630.

Wu, J. \& Weiss, B. (1992). Two-stage induction of the soxRS (superoxide response) regulon of Escherichia coli. J Bacteriol 174, 3915-3920.

Zhang, X. S., Garcia-Contreras, R. \& Wood, T. K. (2007). YcfR (BhsA) influences Escherichia coli biofilm formation through stress response and surface hydrophobicity. J Bacteriol 189, 3051-3062.

Zhao, K., Liu, M. \& Burgess, R. R. (2005). The global transcriptional response of Escherichia coli to induced sigma 32 protein involves sigma 32 regulon activation followed by inactivation and degradation of sigma 32 in vivo. J Biol Chem 280, 17758-17768.

Zheng, M., Wang, X., Templeton, L. J., Smulski, D. R., LaRossa, R. A. \& Storz, G. (2001). DNA microarray-mediated transcriptional profiling of the Escherichia coli response to hydrogen peroxide. J Bacteriol 183, 4562-4570.

Edited by: S. C. Andrews 